\title{
Eyelid problems in general practice
}

\author{
Smit D, MBChB, Dip Ophth(SA), FCOphth(SA), MMed(Ophth), Senior Specialist \\ Department of Ophthalmology, Faculty of Health Sciences, Stellenbosch University, Tygerberg Hospital \\ Correspondence to: Dr Derrick Smit, e-mail: dpsmit@sun.ac.za \\ Keywords: eyelid problems, infections, entropion, ectropion, tumours, blepharitis
}

\begin{abstract}
General practitioners are often confronted with complaints regarding the eyelids. The author presents a number of clinical cases that illustrate common eyelid problems, and provides a discussion of each case to highlight the important features of the condition. Appropriate treatment for each condition is also covered. Many eyelid conditions are amenable to treatment from general practitioners, but those conditions requiring specialist management need to be identified, and referred appropriately.
\end{abstract}

C Medpharm

S Afr Fam Pract 2012;54(3):214-220

\section{Introduction}

Patients of all ages may present at their general practitioners with a variety of eyelid-related complaints. Many eyelid problems may be successfully managed by a family physician, while others might require referral for specialist evaluation and treatment. This quiz includes examples of both scenarios, and aims to emphasise key points in the diagnosis and management of common eyelid conditions.

\section{Case 1}

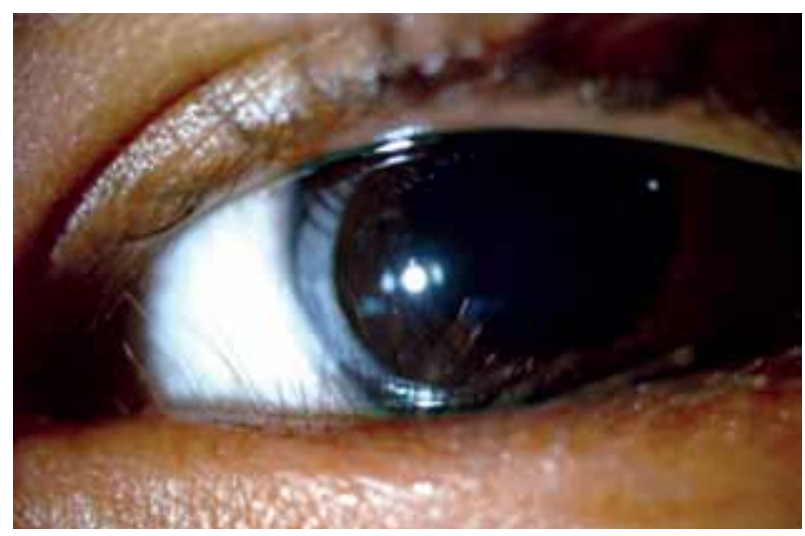

Clinical scenario: A three-year-old girl presents, complaining of a scratchy left eye. Her mother mentions that the eye has been troubling her since she was a few months old, and that the ocular discomfort appears to be worse when she blinks. She has also noticed that the eye is constantly tearing. On examination, her visual acuity is found to be $6 / 6$ in both eyes when tested with a picture card, and her right eye is completely normal. Her left lower lid shows eyelashes that are turned in towards the cornea, and they appear to be scratching the inferior part of the cornea. Examination of the cornea with a cobalt blue light, after the instillation of $2 \%$ fluorescein drops, does not reveal an obvious epithelial defect.

Question: What is the most likely diagnosis, and how would you manage this patient?

\section{Diagnosis: Congenital entropion}

Entropion is a malposition of the eyelid, where the eyelid margin is turned inwards towards the cornea, and most often affects the lower lid. This causes the eyelashes to scratch the cornea, and may result in marked ocular discomfort, constant tearing and even ulceration of the cornea. ${ }^{1}$ Entropion may be congenital or acquired. True congenital entropion is quite rare, and must be distinguished from conditions such as epiblepharon (an extra fold of skin below the eyelid causing the lashes to point superiorly), and trichiasis (posteriorly misdirected lashes), in order to offer the appropriate treatment. Acquired entropion may be either involutional or cicatricial. Involutional entropion occurs in older patients, and results from increased laxity of the eyelid, combined with altered muscle forces, that cause the eyelid to turn inwards. Cicatricial entropion results from scarring on the inner aspect of the eyelid, and is often seen following surgical, chemical, infectious or autoimmune insults to the eyelid, or its conjunctival lining. Frequent use of artificial tears to lubricate the ocular surface, as well as applying a skin plaster to pull the eyelashes outwards, 
may be used to provide temporary relief, but definitive management requires referral to an ophthalmologist to determine the exact underlying cause, and then planning surgical correction accordingly.

\section{Case 2}

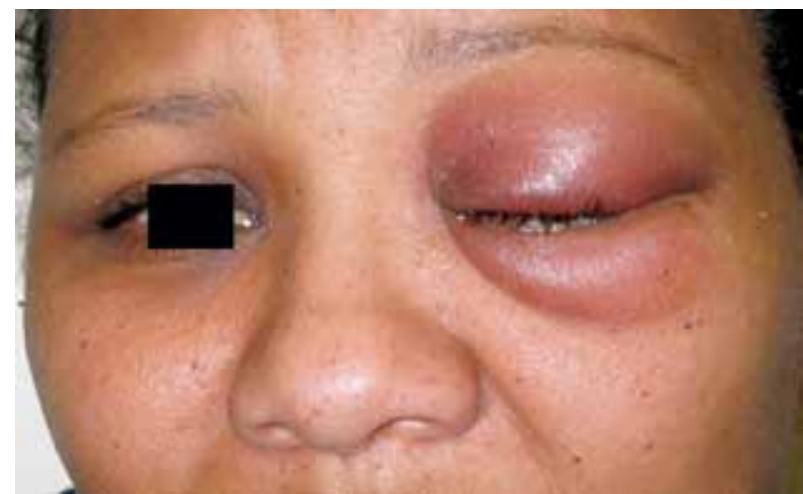

Clinical scenario: A 35-year-old woman presents with a three-day history of painful swelling of her left eyelid. She mentions that she has been feeling unwell for about a week, and has a productive cough. She cannot recall any recent trauma to her eyelids, and maintains that her vision was intact before her eyelid was swollen shut. On examination, her right eye is found to be normal. The left eyelid is warm and tender to touch. After opening the eyelid with a lid speculum, the conjunctiva is found to not be injected, her visual acuity is $6 / 6$, she does not have proptosis or a relative afferent pupil defect, and she is able to move her left eye through a full range of motions without any pain.

Question: What is the most likely diagnosis, and how would you manage this patient?

\section{Diagnosis: Preseptal cellulitis}

Preseptal cellulitis is an infection of the eyelids and soft tissues anterior to the orbital septum, which means that the orbit itself is not affected. The most common causative organisms are Streptococcus pneumoniae, Staphylococcus aureus and Haemophilus influenzae. It often occurs after skin trauma, such as abrasions, lacerations or insect bites, but may also result from the contiguous spread of local infections, such as dacryocystitis or haematogenous spread from more remote infections, i.e. upper respiratory tract infections. Preseptal cellulitis is usually a unilateral condition, and the affected eyelids are red and tender with a variable amount of swelling. ${ }^{2}$

It is very important to be able to distinguish preseptal cellulitis from orbital cellulitis, which is a potentially lifethreatening infection of the orbital contents.

The following signs and symptoms are used to clinically distinguish between the two:
- Visual acuity: Visual acuity is typically unaffected in preseptal cellulitis, but often decreased in orbital cellulitis.

- Pupil reactions: These are normal in preseptal cellulitis, but may reveal a relative afferent pupil defect in orbital cellulitis,

- Chemosis or swelling of the conjunctiva: This is usually absent in preseptal cellulitis, and present in orbital cellulitis.

- Proptosis: This is absent in preseptal cellulitis, but may be mild to marked in orbital cellulitis.

- Eye movements: These are painless and unrestricted in preseptal cellulitis, but may be painful and limited in orbital cellulitis. $^{3}$

Patients with typical preseptal cellulitis may be treated with broad-spectrum oral antibiotics at home, whereas those with suspected orbital cellulitis need to be admitted to hospital for orbital imaging and intravenous antibiotics, preferably under the combined care of an ophthalmologist and ear, nose and throat (ENT) surgeon.

\section{Case 3}

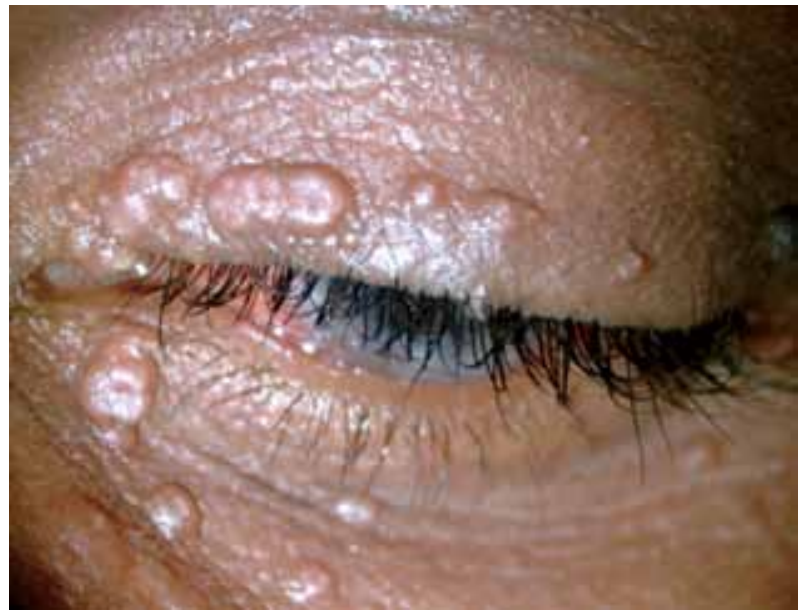

Clinical scenario: A 28-year-old woman presents with a three-month history of discomfort in both eyes. She mentions that the eyes often appear red, and that a watery discharge is present. When looking in the mirror, she has noticed multiple wart-like lesions on her upper and lower lids. She has no previous ocular or medical history of note. On examination, the visual acuity is found to be normal in both eyes. She has multiple waxy, umbilicated nodules on her upper and lower lids, and a few of these are noted to be confluent. The conjunctiva is moderately injected with a marked follicular reaction, evident in the inferior fornices. The corneas are clear and the rest of the examination is within normal limits. 
Question: What is the most likely diagnosis, and how would you manage this patient?

Diagnosis: Molluscum contagiosum infection of the eyelids

Molluscum contagiosum is caused by a DNA poxvirus with an incubation period of six to eight weeks. ${ }^{4}$ Most cases are seen in immunocompetent children, although an increasing number of cases now occur in immunocompromised patients of all ages. In immunocompetent patients, the pale, waxy, umbilicated nodules typically occur unilaterally, and are smaller in size and fewer in number. However, in immunocompromised patients, the nodules tend to be larger and more numerous, often occur bilaterally, and tend to become confluent.

When the nodules are present on the eyelid margin, active virus particles may be shed into the tear film, and give rise to a chronic follicular conjunctivitis. Treatment is often not necessary in immunocompetent patients, since these lesions tend to undergo spontaneous regression. In immunocompromised patients, treatment is usually required, and may take the form of cryotherapy with liquid nitrogen, shave excision, incision and curettage, or cauterisation. The lesions often recur in patients with advanced human immunodeficiency virus (HIV) infection, although the initiation of antiretroviral therapy may lead to complete resolution of disseminated disease. ${ }^{4}$ Therefore, it is important to consider the possibility of compromised immunity in a patient who presents with atypical molluscum contagiosum.

\section{Case 4}

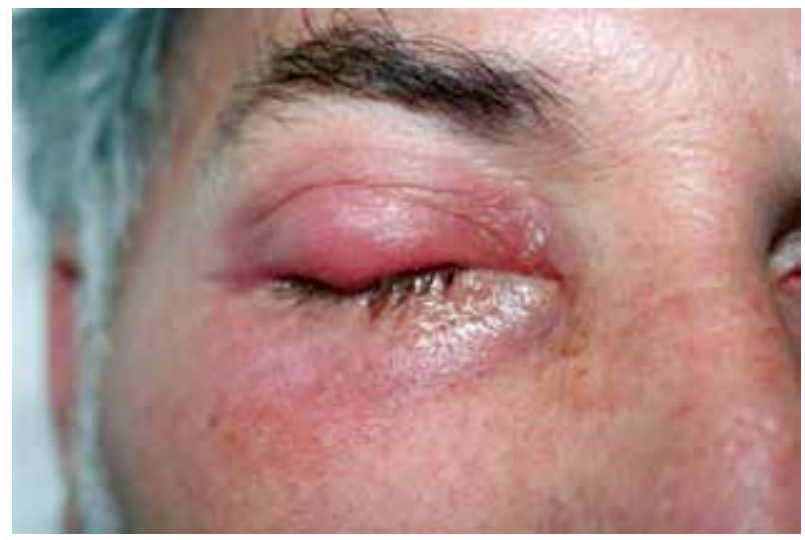

Clinical scenario: A 60-year-old man presents with a fourday history of a painful, swollen right upper lid. He mentions that he developed a sticky discharge in the affected eye about two days before the lid swelling and throbbing pain started. He also gives a history of being aware of a painless pea-sized lump in the upper lid that was present before the current problem started, and that it had gradually increased in size over several months. On examination, his right upper lid is found to be red and swollen. On gentle palpation, a very tender nodule is found. Its location is towards the lateral aspect of the upper lid. Eversion of the upper lid reveals a $10 \times 8 \mathrm{~mm}$ inflamed nodule in the tarsal plate, which appears to fluctuate when pressed upon with a cotton bud. His conjunctiva is mildly injected, but otherwise the rest of the examination is normal.

Question: What is the most likely diagnosis, and how would you manage this patient?

Diagnosis: Internal hordeolum

Hordeolum is the term used to describe infection of the sebaceous glands in the eyelids. Hordeola are considered to be external (styes) when they affect the glands of Zeis at the base of the eyelashes, and internal when the meibomian glands in the tarsal plates are affected. ${ }^{5}$ Most of these infections are caused by Staphylococcus species. In contrast, a chalazion or meibomian cyst, refers to a chronic, sterile, lipogranulomatous lesion that develops when a meibomian gland becomes blocked. When a chalazion becomes secondarily infected, it is then referred to as an internal hordeolum. External hordeola tend to drain spontaneously. Warm compresses and epilation of the affected eyelash may also be considered if speedier resolution is required. However, internal hordeola often require incision and drainage from the conjunctival side once the affected lid has been everted using a special clamp. A course of oral antistaphylococcal antibiotics may also be required.

\section{Case 5}

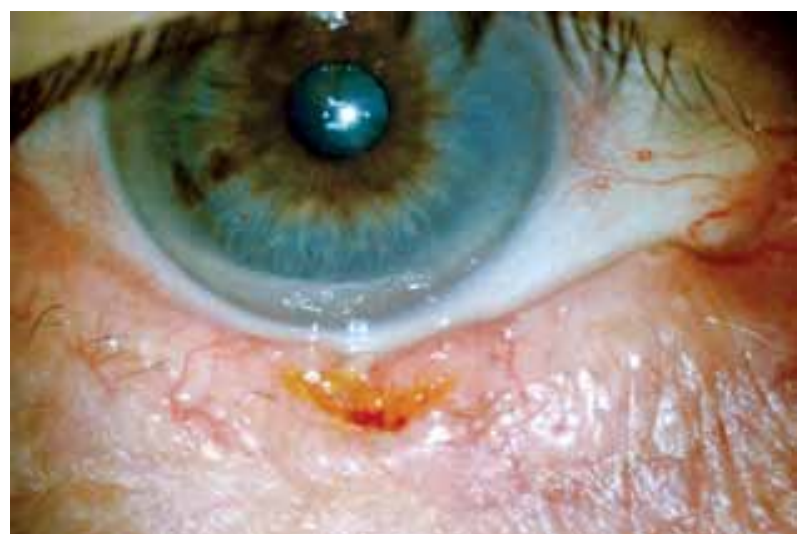

Clinical scenario: A 70-year-old man complains of a sore on his right lower lid that is not healing properly. His history reveals hat he first noticed a smallish lump in the middle of his lower eyelid about two years previously, and that it had enlarged over a period of several months until it eventually formed a raw area in the middle. Despite the use of several different ointments and remedies, he was not able to get it to heal. Examination of the right lower lid reveals a $5 \times 3 \mathrm{~mm}$ 
central ulcer, surrounded by raised, rolled edges containing telangiectatic blood vessels. The eyelashes are noted to be absent in this area. The rest of the eye examination is normal.

Question: What is the most likely diagnosis, and how would you manage this patient?

\section{Diagnosis: Basal cell carcinoma (rodent ulcer)}

Basal cell carcinoma (BCC) predominantly affects elderly patients. It is the most common of all malignancies encountered in humans, and mostly occurs on the head and neck area. Approximately $90 \%$ of malignant eyelid tumours are BCC, and the majority of these occur on the lower lid. ${ }^{6}$ Well-known risk factors include chronic exposure to excessive sunlight, fair skin and smoking. Most small tumours can be successfully excised with a 4-mm margin of clinically normal tissue by a surgeon with appropriate experience in lid surgery. Larger BCC, especially those involving the medial canthus, require more specialised care. Radiotherapy and cryotherapy may also be considered for the treatment of small lesions in patients who are not amenable to surgical excision.

\section{Case 6}

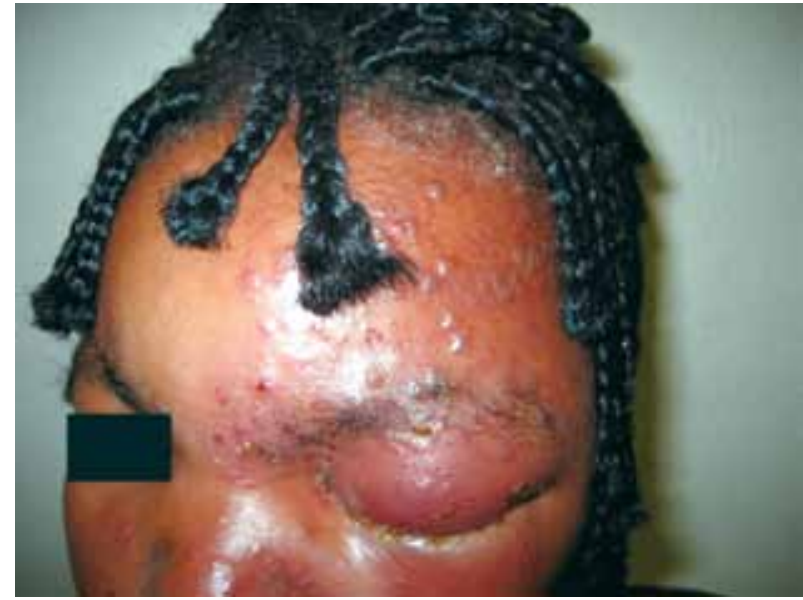

Clinical scenario: A 25-year-old woman complains of an extremely painful rash that has affected her left eyelid, as well as the left side of her forehead, for two days. She mentions that she started feeling generally unwell with flulike symptoms about five days prior to the appearance of the rash, and became aware of a tingling sensation over the left side of her forehead two days later. Her eyelids then began swelling and the tingling sensation was replaced by a deep pain. She also mentions that her vision became blurry before her eyelids closed completely. On examination, she is found to have a vesicular rash over her left forehead and eyelids which is very tender to touch. A few of the lesions have started crusting, and she has similar lesions affecting the left side of her nose. After opening her eyelids with a speculum, her eye shows moderate conjunctival injection, and visual acuity of $6 / 12$. Her pupil reactions are normal, and her cornea does not stain with $2 \%$ fluorescein. The rest of the examination is normal.

Question: What is the most likely diagnosis, and how would you manage this patient?

\section{Diagnosis: Herpes zoster ophthalmicus}

Herpes zoster ophthalmicus (HZO), or ophthalmic shingles, is the result of reactivation of the latent varicella-zoster virus (human herpesvirus 3) in the trigeminal ganglion, which then affects the ophthalmic division of the trigeminal nerve (V1). It represents $10-25 \%$ of all cases of herpes zoster, and used to be a disease that affected the elderly primarily. ${ }^{7}$ However, since the advent of the HIV/ acquired immune deficiency syndrome (AIDS) pandemic, an increasing number of younger patients are also presenting with this condition. The characteristic rash is typically preceded by an influenzalike prodromal phase, which lasts about one week. The dermatomal rash, which always respects the midline, begins with erythematous macules, and progresses rapidly via papules and clear vesicles to pustules, which eventually rupture and form a crust. Hutchinson's sign occurs when the rash involves the tip and ipsilateral side of the nose, and when positive, indicates that the patient has a significantly increased likelihood of ocular involvement on that side. Ocular involvement may take the form of conjunctivitis, episcleritis, keratitis, anterior uveitis, or even retinal necrosis, although cranial nerve palsies have also been reported. ${ }^{7}$ Management of $\mathrm{HZO}$ needs to address three main points: treating the acute viral infection, alleviating the severe associated pain, and preventing postherpetic neuralgia. ${ }^{8}$ Studies have shown that initiation of oral antiviral therapy, within 72 hours of the onset of the rash, alleviates the acute pain, shortens the duration of the rash and reduces ocular involvement from $50 \%$ to $20-30 \% .^{7-9}$ It remains controversial as to whether these drugs prevent or reduce the severity of postherpetic neuralgia. Oral acyclovir (800 mg five times a day) for 7-10 days, valacyclovir (1 000 $\mathrm{mg}$ three times a day) for 7-14 days and famciclovir ( $500 \mathrm{mg}$ three times a day) for seven days are all effective. ${ }^{7-9}$ Some patients may experience adequate pain relief with simple analgesics such as paracetamol, although the vast majority will require the addition of an opioid such as codeine or dextropropoxyphene. Oral corticosteroids have been shown to reduce pain and postherpetic neuralgia, but are not routinely used in $\mathrm{HZO}$ since many patients are already immunocompromised. It is important to remember that any young patient presenting with $\mathrm{HZO}$ should be considered to be potentially immunocompromised, and will require further investigation to either confirm or exclude this possibility. 


\section{Case 7}

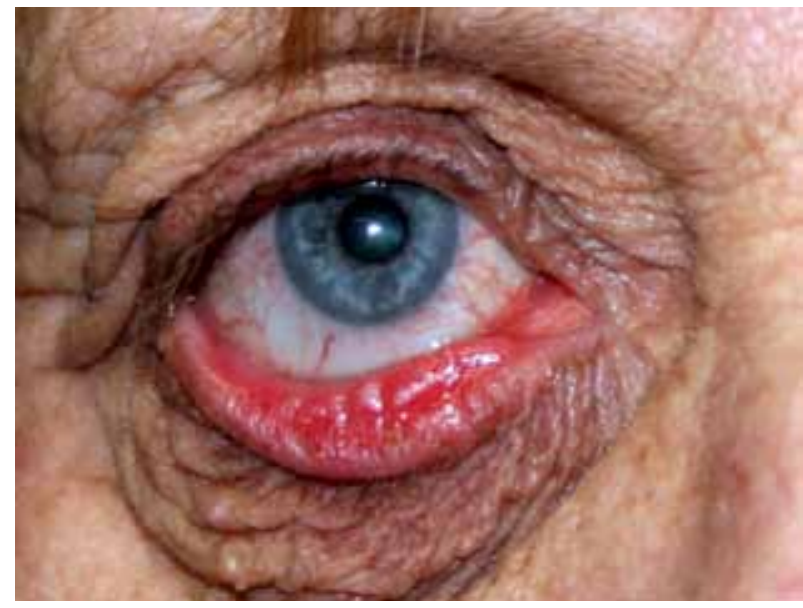

Clinical scenario: A 70-year-old woman complains that her eyes are always watering. She states that she has to dry her eyes constantly as the tears keep running down her cheeks, and she believes that this is causing her lower lids to appear red. She cannot recall when it all started, but is certain that the watering has persisted for years, and is gradually becoming worse. Examination reveals symmetrical thickening and redness of her lower eyelids, and on closer inspection, it is apparent that the eyelashes of both lids are turned out and actually pointing inferiorly. The puncta of the inferior canaliculi on both sides are also not in contact with the eyeball. Gentle traction on the middle part of the lower lid allows the eyelid to be easily pulled more than $10 \mathrm{~mm}$ away from the globe. Apart from early cataracts that are causing a mild decrease in visual acuity, the rest of the examination is normal for both eyes.

Question: What is the most likely diagnosis, and how would you manage this patient?

Diagnosis: Involutional ectropion

Ectropion is a malposition of the eyelid, where the eyelid margin either sags, or is pulled away from its normal position on the surface of the eyeball. The condition may be unilateral or bilateral, depending on the cause. Ectropion may be congenital or acquired. Congenital ectropion is rare, and is often associated with other systemic abnormalities. Acquired ectropion may be subdivided into involutional, paralytic, cicatricial and mechanical causes. ${ }^{1}$ Involutional ectropion is caused by increased laxity of the eyelid tissues, which allows the eyelid margin to sag away from the eyeball. This leads to chronic inflammation and thickening of the conjunctiva lining the exposed inner surface of the lower lid. Paralytic ectropion is caused by facial nerve palsy, while cicatricial ectropion is the result of contracting scar tissue on the eyelid skin that pulls the lid margin away from the globe. Mechanical ectropion occurs when the eyelid margin is mechanically either pulled away, e.g. by the weight of an eyelid mass, or pushed away, e.g. by conjunctival swelling, from the globe. Lower-lid ectropion often leads to exposure of the inferior cornea, which, if not treated properly, may cause corneal ulceration. Temporary treatment consists of lubrication with frequently applied artificial tear drops during the day, and instilling anhydrous liquid lanolin ointment before taping the lids shut at bedtime to protect the cornea. Definitive treatment depends on the underlying cause, and will require referral to a specialist with an interest in oculoplastic surgery to pinpoint the exact aetiology and plan correction accordingly.

\section{Case 8}

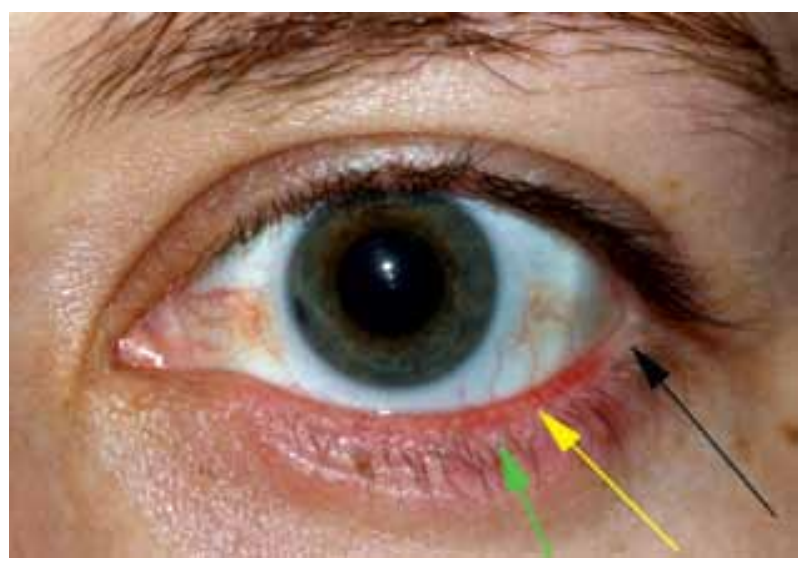

Clinical scenario: A 40-year-old woman complains of bilateral ocular discomfort, which she has felt for a couple of months. She struggles to describe exactly what she is experiencing, and states that at certain times her eyes feel dry and gritty, whereas at other times, there is a burning sensation which occasionally causes her eyes to be sensitive to light. She has also noticed that the discomfort seems to decrease spontaneously for a while, but then usually worsens again. Her case notes show that she consulted a colleague a few months earlier with a meibomian cyst in the left lower lid. On examination, her upper lids appear to be quite normal. Her lower lid margins show marked redness and mild thickening (yellow arrow), when compared to the normal area at the outer canthus (black arrow). On closer inspection, tiny, dandruff-like scales can be seen between her eyelashes (green arrow). The rest of the ocular examination is normal, apart from minimal conjunctival injection in both eyes.

Question: What is the most likely diagnosis, and how would you manage this patient?

\section{Diagnosis: Anterior blepharitis}

Blepharitis is a very common disease which includes various types of inflammation of the eyelid skin, the 
eyelashes and the meibomian glands. It is typically a bilateral, chronic condition that waxes and wanes over time. It may be classified anatomically into anterior blepharitis, which involves the anterior eyelid margin and eyelashes, or posterior blepharitis, which affects the meibomian glands. Anterior blepharitis is often subdivided into staphylococcal, seborrhoeic or mixed forms, while posterior blepharitis usually refers to different forms of meibomian gland disease. ${ }^{10}$ Symptoms are often non-specific, and include burning, tearing, grittiness, foreign body sensation, or even photophobia. Contact lens wearers often develop lens intolerance during disease exacerbations. Signs include redness, crusting, or scarring of the eyelid margin. Small scales may be seen between the eyelashes, or the lashes may be matted together, while in some cases, the tear film may form a frothy layer on the eyelid margin. The mainstay of treatment is meticulous lid hygiene which consists of warm compresses and lid scrubs. Baby shampoo, diluted with cooled, boiled water, is often recommended for use with cotton buds to remove scales and other debris between the eyelashes, although commercial lid scrub preparations are also available. Antibiotic ointment, such as fusidic acid or chloramphenicol, may be applied after the lid scrubs to decrease bacterial colonisation that could exacerbate the inflammation. Topical corticosteroids are sometimes indicated for severe inflammation of the eyelid margin. However, a specialist opinion should be considered once the condition reaches this stage, since more serious ocular sequelae may ensue.

\section{References}

1. Piskiniene R. Eyelid malposition: lower lid entropion and ectropion. Medicina (Kaunas). 2006;42:881-884.

2. Kanski JJ. Clinical ophthalmology: a systematic approach. $6^{\text {th }}$ ed. New York: Elsevier, 2007; p. 175-176.

3. Papier A, Tuttle DJ, Mahar TJ. Differential diagnosis of the swollen red eyelid. Am Fam Physician. 2007;76:1815-1824.

4. Biswas J, Sudharshan S. Anterior segment manifestations of human immunodeficiency virus/acquired immune deficiency syndrome. Indian J Ophthalmol. 2008;56:363-375.

5. Pasternak A, Irish B. Ophthalmic infections in primary care. Clin Fam Pract. 2004;6:19-33.

6. Kanski JJ. Clinical ophthalmology: a systematic approach. $6^{\text {th }}$ ed. New York: Elsevier, 2007; p. 109-111.

7. Shaikh S, Ta CN. Evaluation and management of herpes zoster ophthalmicus. Am Fam Physician. 2002;66:1723-1730.

8. Stankus SJ, Dlugopolski M, Packer D. Management of herpes zoster (shingles) and postherpetic neuralgia. Am Fam Physician. 2000;61:2437-248.

9. Opstelten W, Zaal MJW. Managing ophthalmic herpes zoster in primary care. BMJ. 2005;331:147-151.

10. Jackson WB. Blepharitis: current strategies for diagnosis and management. Can J Ophthalmol. 2008;43:170-179. 